\title{
Serum amyloid $A$ is increased in children with abusive head trauma: a gel-based proteomic analysis
}

\author{
Weimin Gao', Chuanwen Lu', Patrick M. Kochanek ${ }^{2,3}$ and Rachel P. Berger ${ }^{3,4}$
}

BACKGROUND: Abusive head trauma (AHT) is the leading cause of death from traumatic brain injury in infants and young children. Identification of mild AHT (Glasgow Coma Scale score: 13-15) is difficult because children can present with nonspecific symptoms and with no history of trauma.

METHODS: Two-dimensional difference gel electrophoresis combined with mass spectrometry was used to compare the serum protein profile of children with mild AHT and agematched controls. Protein changes were confirmed by western blots. Western blots were performed using serum from children with mild, moderate, and severe AHT to assess the effect of injury severity on protein intensity. The protein identifiedserum amyloid A (SAA) - was then measured by enzymelinked immunosorbent assay.

RESULTS: Using serum from 18 mild AHT cases and 20 controls, there were $\sim 1,000$ protein spots; 2 were significantly different between groups. Both spots were identified as SAA. There was no relationship between protein levels and injury severity. SAA concentrations measured by enzyme-linked immunosorbent assay were increased in cases vs. controls.

CONCLUSION: SAA may be a potential biomarker to identify children with mild AHT who present for medical care without a history of trauma and who might otherwise not be recognized as needing a head computed tomography.

A busive head trauma (AHT) (e.g., shaken baby syndrome) is the leading cause of death from child abuse and the leading cause of death from traumatic brain injury (TBI) in infants and young children (1-7). More than $20 \%$ of TBI and $80 \%$ of all deaths due to TBI in children less than $2 \mathrm{y}$ of age are the result of AHT (8-10). Mortality from AHT is as high as $33 \%$, and significant morbidity is seen in over half of the survivors (11-13).

Early diagnosis of AHT is critical; if the diagnosis is missed, children can be returned to violent home where they can be reinjured or killed (14). Proper diagnosis of AHT can be challenging - the history provided by the caretaker is often unreliable. In the majority of cases, the caretaker who is providing the medical history does not give the physician any history of trauma $(15,16)$. In addition, the physical examination and even neurologic examination can be normal or nonspecific, especially in children with mild injury.

As a result of these clinical challenges, there has been ongoing interest in development of a biomarker or biomarker panel which could be used in the acute care setting to screen for brain injury, much the same way that liver function tests are used to screen for liver injury. In studies that have been published to date $(4,17-20)$, the biomarkers that were used had already been evaluated in adults and/or were available for measurement by commercially available products. Ideally, biomarkers that are specific to infants and young children with brain injury and perhaps to AHT might be identified. The goal of these biomarkers would not be to identify AHT per se but rather to identify the need for a head computed tomography (CT) in an infant or young child without a history of trauma in whom the diagnosis of TBI might not otherwise be considered.

The objective of the present study was, therefore, to determine whether there were differences in the serum proteome of infants and young children with mild AHT-defined as a Glasgow Coma Scale (GCS) score of 13-15-compared with age-matched controls. To achieve this objective, proteomic profiles were performed and compared between children with AHT and controls using two-dimensional difference gel electrophoresis (2D-DIGE) coupled with mass spectrometry (MS) analysis. The protein(s) of interest were further confirmed by western blot. Finally, the clinical implication of the candidate protein(s) was evaluated by measurement using an enzyme-linked immunosorbent assay (ELISA). This is a similar approach to the one we used to compare the cerebrospinal fluid proteome between children with severe AHT and those with severe noninflicted TBI (21). In that study, there were differences in the cerebrospinal fluid concentrations of cystatin C (CC), haptoglobin (HP), and prostaglandin-D synthase (PGDS) between children with severe AHT and those with severe noninflicted TBI.

\section{RESULTS}

\section{D-DIGE and Protein Identification}

The initial 2D-DIGE was performed as described above using pooled serum from 38 subjects (20 controls and 18 cases, 
Proteomic analysis

38 Samples: 18 cases with mild abusive head trauma/20 age-, gender-, and race-matched CT negative controls

Identification of spots which differ between groups

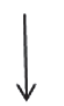

Mass spectrometry identified serum amyloid A (SAA)

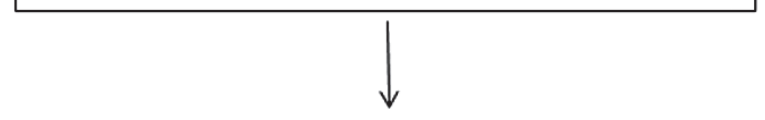

Western blot analysis \#1 performed on 38 samples from initial proteomic analysis

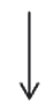

Western blot analysis \#2 performed on 20 unique samples from children with mild $(n=10)$ and moderate/severe $(n=10)$ abusive head trauma

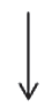

Serum SAA concentrations measured by ELISA in 96 unique subjects (61 cases and 35 controls)

Figure 1. Study design. ELISA, enzyme-linked immunosorbent assay.

group 1; Figure 1). There was no difference in the mean (SD) age (in months) $(6.0(6.6)$ vs. $4.9(5.2))$, the proportion of males ( 44 vs. $45 \%$ ), or the proportion of Caucasians ( 75 vs. $67 \%)$ between cases and controls. For the cases, the serum samples were collected a mean (SD) of 13.4 (12.2) h after injury. All 18 cases had acute extra-axial hemorrhage; 12 cases $(67 \%)$ also had evidence of intraparenchymal injury, and $8(44 \%)$ had a skull fracture. In 4 cases $(22 \%)$, there was concern for previous injury. Five of the 18 cases $(28 \%)$ had extracranial fractures. Liver function tests were measured in 15 cases (83\%), and 2 subjects had increased liver function tests and underwent an abdominal CT that did not demonstrate abdominal injury.

Approximately 1,000 protein spots were detected. Duplicate DIGE gels with reverse label were run and showed remarkably high between-gel reproducibility ( $>98 \%)$. A representative DIGE gel is shown in Figure 2. From these detected spots, two spots with the highest changes ( $>3$-fold) were analyzed by liquid chromatography (LC)/MS/MS for protein identification. These two spots were both identified as serum amyloid A (SAA) (eight unique peptides with 59\% sequence coverage for one spot and nine unique peptides with $64 \%$ sequence coverage for the other spot).

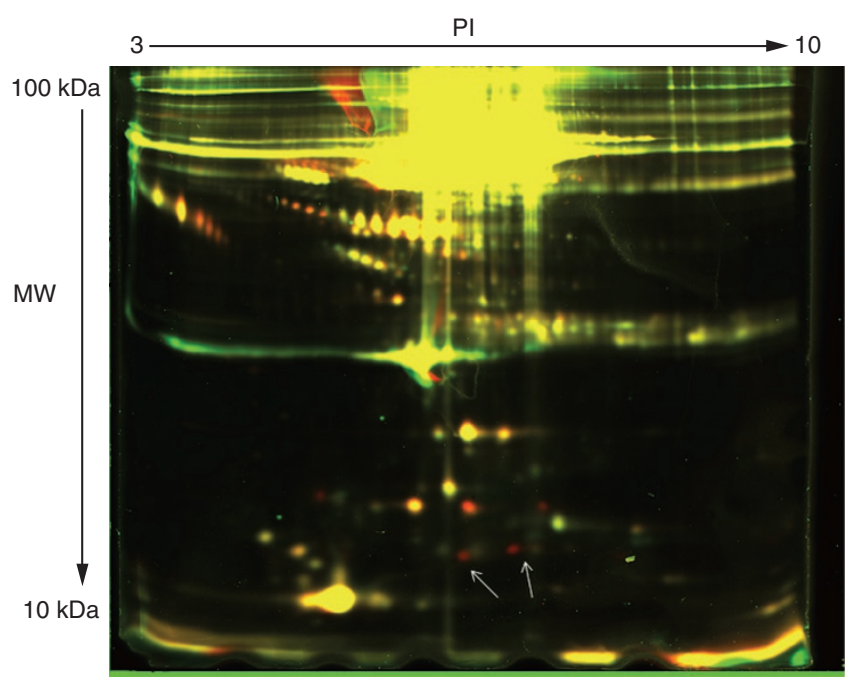

Figure 2. A representative composite two-dimensional difference gel electrophoresis of AHT and control. Serum samples from cases (e.g., patients with mild AHT) and controls were labeled with Cy5 and Cy3, respectively. Internal control using both cases and controls was labeled with Cy2. Yellow spots indicate that the protein concentrations were the same in cases and controls. Red spots indicate that the protein concentrations were higher in cases, and the green spots indicate that the protein levels were higher in controls. AHT, abusive head trauma.

\section{Western Blot Analyses of SAA, CC, HP, and PGDS}

Western blot analysis was performed to measure the SAA expression from the 38 individual samples analyzed previously by $2 \mathrm{D}-\mathrm{DIGE}$. The mean (SD) expression of SAA was significantly higher in cases vs. controls $(1,213.1$ (804.5) vs. 203.0 (221.0) units; $P<0.01$; Figure 3a,b). There was no relationship between age and mean (SD) SAA concentrations in either cases or controls. There was also no relationship between the mean expression of SAA in cases and the presence or absence of fractures. Figure $3 \mathrm{c}$ shows the receiver operating characteristic (ROC) curve that was obtained with these 38 subjects; the area under the ROC curve was 0.96 (95\% confidence interval: $0.90-1.00)$.

Western blots were also performed on the 38 samples to measure CC, HP, and PGDS expression because these three proteins had been identified by our previous gel-based proteomic study for cerebrospinal fluid in children with severe AHT (21). PGDS was not detectable in either cases or controls. $\mathrm{CC}$ and HP were detectable in both cases and controls, but without any difference between groups (Figure 3a).

\section{Assessment of SAA Expression Along the Injury Severity Spectrum Using Western Blots}

To assess whether SAA expression was related to injury severity, the western blots were repeated using 20 unique serum samples from children with mild AHT (initial GCS score: $13-15 ; n=10)$ and children with moderate-to-severe AHT (initial GCS score: $3-12 ; n=10$; group 2). There was no difference in the mean (SD) age (15.1 (14.4) vs. 12.9 (12.4) mo) or in the time after injury when SAA was measured (12.0 (8.1) 
a

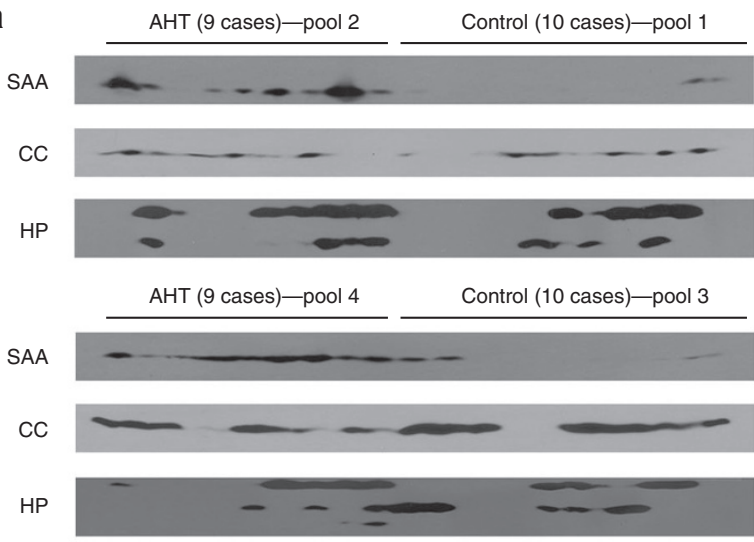

b

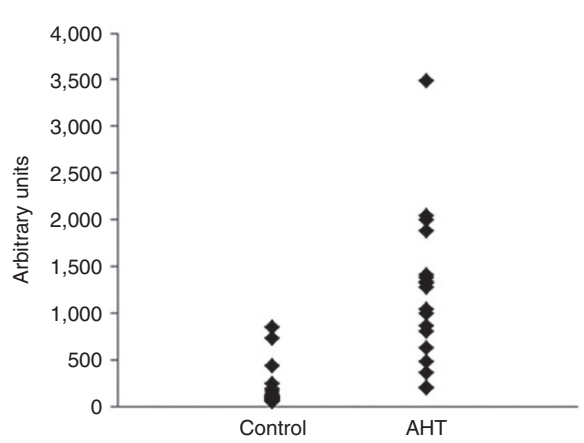

C

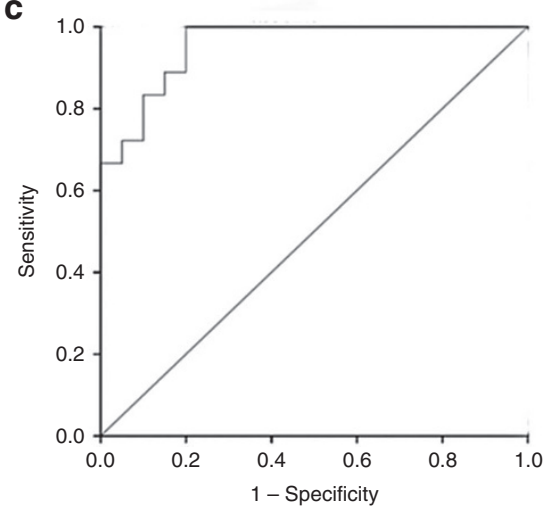

Figure 3. Levels of serum amyloid $A(S A A, 14 \mathrm{kDa})$, cystatin $\mathrm{C}(\mathrm{CC}, 13 \mathrm{kDa})$, haptoglobin ( $\mathrm{HP}, 15-18 \mathrm{kDa}, 45 \mathrm{kDa})$, and prostaglandin-D synthase (PGDS, $21 \mathrm{kDa}$ ) in serum from individuals with mild AHT compared with controls. (a) The western blot images of SAA, CC, and HP in cases with mild AHT compared with controls. (b) The protein intensities of SAA in cases with mild AHT compared with controls. The intensity of each band was quantified using Quantity One software (Bio-Rad). (c) Receiver operating characteristic curve of SAA. AHT, abusive head trauma.

vs. $11.7(10.0)$ h) between subjects with mild vs. moderateto-severe AHT. Liver function tests were measured in all 20 subjects and were abnormal in 8 subjects (40\%). Abdominal CT was completed in five of these subjects and was normal in each case. The three subjects who did not undergo abdominal CT had a borderline increase in liver function tests with only aspartate aminotransferase or alanine aminotransferase (not both) slightly above $200 \mathrm{IU} / \mathrm{l}$. There was also no difference in the mean (SD) SAA levels $(4,554.0(3,675.4)$ vs. $6,278.9$ $(6,011.5)$ units) between subjects with mild AHT (initial GCS score: 13-15) and those with moderate-to-severe AHT (initial GCS score: 3-12; Figure 4a,b). Although there was no difference in SAA concentrations based on injury severity, there was variability in SAA concentrations with a range of 1,236-21,989 units. There was no association between time after injury, GCS score, age, the presence or absence of skull fracture, the presence or absence of extracranial fracture, the presence or absence of parenchymal injury, and SAA concentrations.

\section{Serum Amyloid A Enzyme-Linked Immunosorbent Assay}

SAA concentrations were measured by ELISA in 96 subjects (61 AHT cases and 35 controls, group 3). There was no difference in the proportion of cases and controls with respect to gender and ethnicity. The mean (SD) age of cases was younger than controls

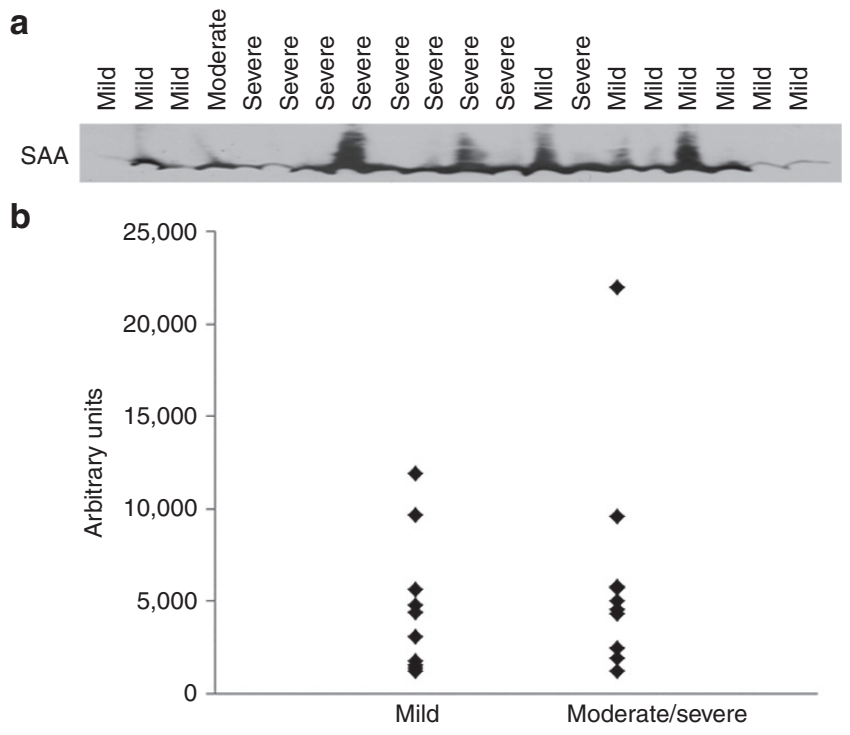

Figure 4. Levels of SAA in individual serum samples from cases with mild (GCS score: 13-15), moderate (GCS score: 8-12), and severe AHT (GCS score: $<8)$. (a) The western blot image of SAA. (b) The protein intensities of SAA in cases with mild, moderate, and severe AHT. The intensity of each band was quantified using Quantity One software (Bio-Rad). Cases were not grouped by severity due to blind experiment. AHT, abusive head trauma; GCS, Glasgow Coma Scale; SAA, serum amyloid A. 


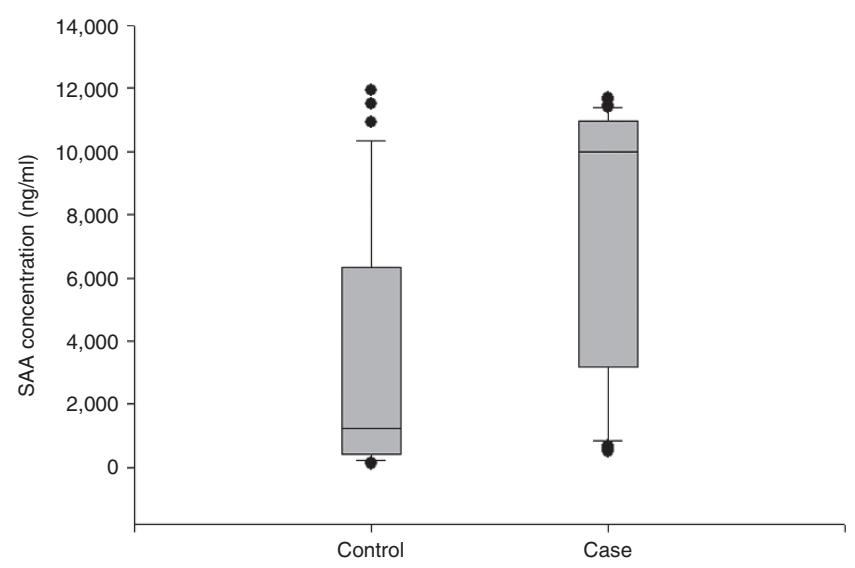

Figure 5. Serum amyloid A (SAA) concentrations measured by enzymelinked immunosorbent assay in cases (children with abusive head trauma) compared with controls. SAA concentrations are measured in nanograms per milliliter. The box values range from 25 to 75 percentile of the total samples, the line within it indicating the median value.

((29.2 (35.0) vs. $50.4(48.0) \mathrm{mo} ; P=0.03)$ ). The mean (SD) initial GCS score was 10.0 (5.0). The mean (SD) time after injury when samples were collected for AHT subjects was 9.2 (8.2) h. Mean (SD) SAA concentrations were higher in cases vs. controls $(7,927$ $(4,165)$ vs. $3,470(3,984) \mathrm{ng} / \mathrm{ml} ; P<0.001$; Figure 5$)$. The area under the ROC curve obtained from these 96 subjects was 0.78 (95\% confidence interval: $0.68-0.88$ ). There was no relationship between age and SAA concentrations in cases or controls, and no relationship between GCS and SAA concentrations.

\section{DISCUSSION}

This is the first study to compare the serum proteomes of children with AHT and a cohort of age-matched controls without brain injury. Our 2D-DIGE followed by MS demonstrated that SAA was increased in children with mild AHT. This increase was confirmed by western blots and subsequently by ELISA. Interestingly, we identified two different protein spots by MS; these spots had similar molecular weights but different $\mathrm{pKa}$, suggesting that there were different forms of SAA present in the samples. This is in consistent with a study published by Bruun $e t$ al. (22), who demonstrated that different isoforms of SAA could be potentially used as biomarkers of neonatal infection.

SAA, an acute-phase reactant, is an apolipoprotein that is involved in the chemotactic recruitment of inflammatory cells to the site of inflammation. It is not surprising that our proteomic analysis would identify an inflammatory marker such as SAA. The role of inflammatory markers in both TBI (23) and cerebral ischemia is well documented (24). SAA has been identified previously as a potential biomarker of injury and prognosis in studies of both TBI and hypoxic-ischemic encephalopathy (HIE). A study by Hergenroeder et al. (25) comparing the proteome of 11 adults with severe TBI to 11 age-, sex-, and race-matched volunteers identified SAA as well as two other proteins-c-reactive protein and retinol-binding protein-as unique to TBI subjects. In a study of adults with ischemic stroke, SAA concentrations and c-reactive protein and interleukin- 6 were significantly higher in patients who died compared with those who survived and were independently associated with early death, after adjusting for various confounding factors (24). In a recent study of SAA in neonatal HIE, SAA concentrations were significantly increased in cases compared with controls both at days 1 and 7 after injury. SAA concentrations at day 1 correlated significantly with the severity of HIE (mild, moderate, and severe) as measured by the Sarnat and Sarnat system (26). Interestingly, the importance of hypoxemia and ischemia in the pathophysiology of AHT has been highlighted in radiologic $(27,28)$, clinical (29), pathologic (30), and biochemical studies (20).

It important to recognize, however, that based on the current study, it is not possible to state that SAA is specific to AHT, only that it discriminates children with AHT from age-matched children with the same clinical symptoms, but without brain injury. We did not evaluate whether the increase in SAA occurs in noninflicted TBI. It is also not possible to be sure that the source of the SAA is the brain. We did not, however, see an association between SAA concentrations and the presence of fracturesskull fractures or extracranial fractures-suggesting that the fractures are not a source of SAA. In addition, almost all children with AHT were screened for abdominal injury with liver function tests, and none had evidence of either hypoxic or traumatic liver injury suggesting that hepatocyte injury was not the source of SAA. While there are other recognized causes of increased serum SAA including infection, cancer, and coronary artery disease $(31,32)$, cancer and coronary artery disease are extraordinarily rare in a pediatric patient population, particularly in a population of infants and toddlers, and there was no clinical evidence of infection in any subject. In fact, having a fever was an exclusion criterion. As discussed previously, the clinical role of a potential biomarker such as SAA would be to provide physicians with an indication that an infant with soft neurologic signs such as vomiting might require a head CT to evaluate for possible brain injury. In this clinical scenario, SAA may be able to act as a biomarker of possible brain injury whether it is released peripherally or centrally. What is important is not the source of SAA (e.g., central or peripheral), but the fact that the increase in SAA occurs in infants with brain injury and not in age-matched children who presented to the emergency department for evaluation of the same symptoms but do not have brain injury. This suggests that being sick, per se, does not result in increased SAA concentrations.

The ability of SAA to discriminate children with AHT from age-matched controls was extremely good as seen by the area under the ROC curve. Indeed, the ability of SAA to discriminate between children with AHT and controls is at least good if not better than serum brain biomarkers that have been extensively studied in pediatric TBI. In a study evaluating the use of serum neuron-specific enolase (28), S100B, and myelin-basic protein to discriminate children with TBI from controls, the AUC for these biomarkers were $0.85,0.82$, and 0.69 (33). In a 2008 study, Lo et al. (6) demonstrated almost identical AUC for neuron-specific enolase $(\mathrm{AUC}=0.83)$ and $\mathrm{S100B}(\mathrm{AUC}=0.83)$ when evaluating the ability of these biomarkers to predict outcome in a cohort of 28 children with severe TBI. These authors have also demonstrated a close to perfect AUC using a combination of biomarkers. 
In contrast to the study by Aly et al. (26), we did not demonstrate an association between injury severity as measured by GCS score either by western blots or ELISA. In that study, SAA concentration in 27 neonates with HIE were compared with SAA concentrations in 27 neonates with HIE of varying severities. The lack of association in our study could be due to the way in which we evaluated injury severity using initial GCS; the lack of sensitivity of the GCS score in infants and young children has been identified previously as a concern (34). Alternatively, the difference between these study populations could be due to the differences in the pathophysiology and timing of neonatal HIE compared with AHT.

One of the limitations of serum biomarkers has been the short serum half-life. S100B, for example, has a half-life of less than $60 \mathrm{~min}$. As a result, increases in $\mathrm{S100B}$ are rarely seen more than $12 \mathrm{~h}$ after injury except in cases of severe TBI (35). While there are no published data related to the serum half-life of SAA in patients with TBI, in a study of adults with pneumonia, the half-life of SAA was 34.9 (28.7) h (36). Although the disease process in pneumonia is clearly different than that in TBI, these data suggest that SAA has a longer half-life than S100B and therefore could be useful even in children whose TBI was more than $12 \mathrm{~h}$ prior to the blood draw. The longer half-life of SAA compared with S100B is supported by the data from the current study cohort in which SAA was still increased in virtually every sample even though the serum samples were collected a mean (SD) of 13.4 (12.2) h after injury. While we did not measure serial SAA concentrations in the current study, in the study of neonatal HIE by Aly et al. (26), SAA remained increased in cases compared with controls up to $7 \mathrm{~d}$ after injury, the last time point measured in the study. Although the prolonged increase in SAA could be due to a prolonged halflife, it is more likely a result of continued release of SAA and/or a combination of the two. While there are clear differences in the pathophysiology of neonatal HIE and AHT, these data suggest that SAA might be detectable for many days after injury.

We did not find a relationship between SAA concentrations and age. Whether or not there is a relationship between age and SAA is inconsistent throughout the literature. Several studies have reported a positive correlation with age in neonates/infants with suspected infections (22) and in healthy adults (37-39), while others have shown no relationship $(40,41)$. Since the increased SAA concentrations may be affected by numerous clinical factors that we did not adjust for (e.g., developmental variation such as premature vs. full-term infants, the presence or absence of chronic subdural hematoma or other extracranial injuries), further validation studies with increased sample size are needed to understand these potential confounders in the pediatric population. It might initially be surprising that we did not identify proteins that have been previously shown to be increased after AHT such as neuron-specific enolase or S100B. This likely relates to our technique; 2D-DIGE used in our study, although with a high sensitivity and accuracy, is a low-throughput technique and not able to provide the analysis of extremely acidic, basic, hydrophobic, smaller $(<10 \mathrm{kDa})$, or low-abundant proteins such as neuronspecific enolase, S100B, GFAP, and MBP.
In summary, using 2D-DIGE followed by MS, western blots, and ELISA, we identified SAA as a unique protein that differed between infants with brain injury and those with routine childhood illness. This increase was not dependent on the severity of the brain injury. These data suggest that SAA might be able to be used as a biomarker to identify infants with mild AHT who present for medical care with soft neurologic signs. These are infants whose brain injury might otherwise be missed and who would benefit from further evaluation with a head CT or brain magnetic resonance imaging (MRI).

\section{MATERIALS AND METHODS \\ Subjects and Serum Samples}

All serum samples were part of a repository of serum samples that is maintained at the Safar Center for Resuscitation Research at the University of Pittsburgh and were stored at $-70{ }^{\circ} \mathrm{C}$. The serum samples in the repository were obtained from multiple studies, all of which were approved by the Institutional Review Board of University of Pittsburgh. Consent was obtained from all controls. An Institutional Review Board-approved waiver of informed consent was used for children with AHT. In each case, AHT was diagnosed by the Children's Hospital of Pittsburgh of UPMC Child Protection Team. Defining AHT based on the evaluation of a hospital-based Child Protection Team is commonly used in a research setting and the definition is the same which we and others have used previously $(10,20,42)$. The physicians on the Child Protection Team perform an evaluation by obtaining a complete medical history, performing a physical examination, and reviewing the results of the neuroimaging (head CT and/or brain MRI), skeletal survey, dilated eye examination, relevant laboratory data, and any other evaluations. They then use their expertise to assess whether the injuries identified are consistent with the history provided by the caretakers of how the injuries occurred. The Child Protection Team is a hospital-based child abuse team and is not affiliated with local Child Protective Services. In every case of AHT, the neuroimaging (CT and/or MRI) was abnormal.

For each serum sample in the repository, there is associated clinical data collected including demographic information, reason for presentation to the hospital, initial GCS score, results of initial head CT and/or brain MRI, and presence or absence of noncranial fractures. The results of the initial head CT and/or brain MRI were classified by the presence or absence of a skull fracture as well as the presence or absence of extra-axial hemorrhage and the presence or absence of intraparenchymal injury. If a concern was raised about the possibility of previous brain injury (e.g., in subjects with subdural hemorrhage of more than one age), this was documented as a dichotomous variable (e.g., presence or absence of concern for previous injury). Subjects with AHT either presented to the hospital because of a history of head trauma or for evaluation of a neurologic symptom (e.g., vomiting, fussiness, or seizures) without a history of trauma. Time of injury for cases was defined as the last time at which the child was asymptomatic. This is a definition we have used consistently in our studies of biomarkers in pediatric TBI $(4,20)$. Extracranial fractures were defined by the results of the skeletal survey that was performed in all cases of AHT and were classified as either "present" or "absent." Liver function tests were used to screen for liver injury in children with AHT. Abnormal liver function tests were defined as $>200 \mathrm{IU} / 1$ for either aspartate aminotransferase or alanine aminotransferase.

There were three groups of cases used for this study: group 1 were children with AHT and a GCS score of 13-15 which were used for the 2D-DIGE and initial western blots; group 2 were children with AHT and GCS score of 3-15 which were used to validate the findings on the initial western blots and to assess the relationship between SAA and injury severity; and group 3 were children with AHT and GCS score of 3-15 that were used to measure SAA by ELISA (Figure 1).

There were two unique groups of controls used for this study in order to provide a comparison group for case group 1 and case group 3. There were no controls corresponded with group 2 since that group was used to evaluate the relationship between injury severity 
and SAA expression. All controls for group 1 presented for evaluation of a neurologic symptom (e.g., vomiting, fussiness, or seizures) without a history of trauma. Group 1 controls were matched to cases by age within 1 mo, by gender, and by race (white vs. nonwhite). These controls either had a normal head CT performed as part of clinical care or did not have a head CT performed because it was not considered clinically necessary. The subgroup who did not have a CT performed as part of clinical care were followed up by phone contact and chart review until 6 mo or 1 y of age after enrollment (which ever came later) and were not subsequently diagnosed with brain injury or child abuse (cranial or noncranial). Group 3 controls included children aged $<1$ y who presented for evaluation of a neurologic symptom, healthy children over the age of $1 \mathrm{y}$ who were having blood collected as part of well child care and children between the ages of 1 and $12 \mathrm{y}$ who presented to the emergency department for a noncranial injury (e.g., fracture) without any evidence of head injury and without concern for child abuse. Group 3 controls were matched to group 3 cases by gender and race.

\section{Two-Dimensional Difference Gel Electrophoresis}

Serum samples from 20 controls and 18 cases were used for this part of study (group 1). Serum samples from 20 control subjects were pooled in two separate control samples and 18 case subjects were pooled in two separate case samples. Therefore, a total of four pooled serum samples were prepared, two each of controls and AHT patients. Samples were labeled following the protocol described previously by our group (21). In brief, serum from cases and controls were reversely labeled with Cy3 and Cy5, respectively (GE Healthcare, Piscataway, NJ). Cy2 was used to label the internal control. Tubes containing $150 \mu \mathrm{g}$ of pooled sample were combined with $1 \mu \mathrm{l}$ of diluted Cy3 or Cy5 (400 pmol/ $\mu \mathrm{l}$ in N,Ndimethylformamide; Sigma, St Louis, MO). After centrifugation, the mixture was left on ice for $30 \mathrm{~min}$ without light exposure. Then, the reaction was stopped by the addition of $1 \mu \mathrm{l}$ of $10 \mathrm{mmol} / \mathrm{l}$ lysine (Sigma) and placement of samples on ice for $10 \mathrm{~min}$ in the dark. Samples labeled with Cy dyes were mixed and diluted to $300 \mu \mathrm{l}$ by adding $2 \mathrm{D}$ rehydration buffer (Bio-Rad, Hercules, CA) consisting of $8 \mathrm{~mol} / \mathrm{l}$ urea, $0.5 \%$ 3-((3-Cholamidopropyl)dimethylammonio)-1-propanesulfonate, 10 $\mathrm{mmol} / \mathrm{l}$ dithiothreitol (Bio-Rad), $0.2 \%$ biolytes ampholyte, and trace bromophenol blue. Samples were then applied to $17-\mathrm{cm}$ immobilized linear $\mathrm{pH}$ 3-10 gradient (IPG) strips (Bio-Rad) for overnight rehydration. Isoelectric focusing was conducted at $250 \mathrm{~V}$ for $20 \mathrm{~min}$, gradually increased to 10,000 within $2.5 \mathrm{~h}$, and held at $10,000 \mathrm{~V}$ for a total of 50,000 Voltage hours (Vh). IPG strips were subsequently equilibrated with buffer I ( $6 \mathrm{~mol} / \mathrm{l}$ urea, $2 \%$ sodium dodecyl sulfate, $375 \mathrm{mmol} / \mathrm{l}$ Tris-HCl (pH 8.8), 20\% glycerol, $130 \mathrm{mmol} / \mathrm{l}$ dithiothreitol, and trace bromophenol blue) and buffer II ( $6 \mathrm{~mol} / \mathrm{l}$ urea, $2 \%$ sodium dodecyl sulfate, $375 \mathrm{mmol} / \mathrm{l}$ Tris-HCl, $20 \%$ glycerol, $135 \mathrm{mmol} / \mathrm{l}$ iodoacetamide, and trace bromophenol blue). Proteins were then separated with 12\% sodium dodecyl sulfate-polyacrylamide gel electrophoresis and visualized using a Typhoon Trio Imager (GE Healthcare) at excitation wavelengths of 488, 532, and $633 \mathrm{~nm}$ for $\mathrm{Cy} 2, \mathrm{Cy} 3$, and Cy5, respectively. Images were manipulated and analyzed by DeCyder (GE Healthcare Life Sciences, Piscataway, NJ) and ImageQuant software (Topcon Positioning Systems, Livermore, CA); protein intensity differences were calculated for each spot on every gel.

\section{In-Gel Digestion and LC/MS/MS Identification}

Spots of interest were isolated using a spot picker, and placed into a $0.5 \mathrm{ml}$ Eppendorf tube for trypsin digestion as described previously $(21,43)$. Trypsinized peptides were analyzed by nano LC/MS/MS on a Thermo Fisher LTQ Orbitrap XL (Thermo Scientific, Waltham, MA). MS results were searched using Mascot (Matrix Science, Boston, MA). Samples were processed in the Scaffold algorithm using DAT files generated by Mascot. Parameters for LTQ Orbitrap XL data require a minimum of two peptide matches per protein with minimum probabilities of $90 \%$ at the protein level.

\section{Western Blot Analyses}

Serum concentrations of SAA, CC, HP, and PGDS in individual serum samples from cases and controls were analyzed by western blots as described previously (44). Briefly, $5 \mu \mathrm{l}$ of serum sample was separated by $12 \%$ sodium dodecyl sulfate-polyacrylamide gel electrophoresis and then transferred to polyvinylidene fluoride membranes. The immobilized proteins were then incubated overnight at $4{ }^{\circ} \mathrm{C}$ in blocking buffer containing $3 \%$ nonfat dry milk in $1 \times$ phosphate-buffered saline (PBS) and $0.1 \%$ Tween 20 (1× PBST). After blocking, the membranes were probed with the primary antibody for $1 \mathrm{~h}$. Antibody binding was detected with horseradish peroxidase-conjugated secondary antibody at a dilution of 1:1,000 for $1 \mathrm{~h}$ at room temperature. After a brief incubation with enhanced chemiluminescence, the signals on membranes were exposed to X-ray films. Relative densitometric digital analysis of protein bands were determined using Quantity One software (Bio-Rad).

\section{SAA ELISA Assay}

The SAA ELISA was purchased from US Biological (Swampscott, MA) and run according to manufacturer's instructions. The lower limit of detection of the ELISA is $4 \mathrm{ng} / \mathrm{ml}$.

\section{Statistical Analyses}

For 2D-DIGE, image analysis was carried out with DeCyder software and ImageMaster software. For DeCyder software, the differential ingel analysis module was used to process a pair of images from a single gel and perform spot detection and quantification. The biological variation analysis was employed to calculate ratios between samples and controls by performing a gel-to-gel matching of the pair of spot maps from each gel. One-way ANOVA was used to analyze the difference of protein levels detected by western blots or ELISA. ROC curve was generated to assess the accuracy of SAA in predicting AHT. All analyses were performed using SPSS software (SPSS, Chicago, IL), and differences with $P<0.05$ were considered statistically significant.

\section{STATEMENT OF FINANCIAL SUPPORT}

This work was supported by National Institutes of Health grant 1R01HD055986 (Bethesda, MD).

Disclosure: The authors declare no conflict of interest.

\section{REFERENCES}

1. Childhood injuries in the United States. Division of Injury Control, Center for Environmental Health and Injury Control, Centers for Disease Control. Am J Dis Child 1990;144:627-46.

2. Duhaime AC, Christian CW, Rorke LB, Zimmerman RA. Nonaccidental head injury in infants-the "shaken-baby syndrome". N Engl J Med 1998;338:1822-9.

3. Adelson PD, Kochanek PM. Head injury in children. J Child Neurol 1998;13:2-15.

4. Berger RP, Tảasan S, Rand A, Lokshin A, Kochanek P. Multiplex assessment of serum biomarker concentrations in well-appearing children with inflicted traumatic brain injury. Pediatr Res 2009;65:97-102.

5. Fountas KN, Tasiou A, Kapsalaki EZ, et al. Serum and cerebrospinal fluid C-reactive protein levels as predictors of vasospasm in aneurysmal subarachnoid hemorrhage. Clinical article. Neurosurg Focus 2009;26:E22.

6. Lo TY, Jones PA, Minns RA. Pediatric brain trauma outcome prediction using paired serum levels of inflammatory mediators and brain-specific proteins. J Neurotrauma 2009;26:1479-87.

7. Murphy S. Pediatric neurocritical care. Neurotherapeutics 2012;9:3-16.

8. Duhaime AC, Alario AJ, Lewander WJ, et al. Head injury in very young children: mechanisms, injury types, and ophthalmologic findings in 100 hospitalized patients younger than 2 years of age. Pediatrics 1992;90(2 Pt 1):179-85.

9. Goldstein B, Kelly MM, Bruton D, Cox C. Inflicted versus accidental head injury in critically injured children. Crit Care Med 1993;21:1328-32.

10. Keenan HT, Runyan DK, Marshall SW, Nocera MA, Merten DF, Sinal SH. A population-based study of inflicted traumatic brain injury in young children. JAMA 2003;290:621-6.

11. Herman BE, Makoroff KL, Corneli HM. Abusive head trauma. Pediatr Emerg Care 2011;27:65-9.

12. Sieswerda-Hoogendoorn T. Several neuroradiological features can help distinguish abusive and non-abusive head trauma. J Pediatr 2012;160: $880-1$. 
13. Spaide RF, Swengel RM, Scharre DW, Mein CE. Shaken baby syndrome. Am Fam Physician 1990;41:1145-52.

14. Jenny C, Hymel KP, Ritzen A, Reinert SE, Hay TC. Analysis of missed cases of abusive head trauma. JAMA 1999;281:621-6.

15. Ettaro L, Berger RP, Songer T. Abusive head trauma in young children: characteristics and medical charges in a hospitalized population. Child Abuse Negl 2004;28:1099-111.

16. Hettler J, Greenes DS. Can the initial history predict whether a child with a head injury has been abused? Pediatrics 2003;111:602-7.

17. Berger RP, Adelson PD, Richichi R, Kochanek PM. Serum biomarkers after traumatic and hypoxemic brain injuries: insight into the biochemical response of the pediatric brain to inflicted brain injury. Dev Neurosci 2006;28:327-35.

18. Berger RP, Beers SR, Richichi R, Wiesman D, Adelson PD. Serum biomarker concentrations and outcome after pediatric traumatic brain injury. J Neurotrauma 2007;24:1793-801.

19. Berger RP, Hayes RL, Richichi R, Beers SR, Wang KK. Serum concentrations of ubiquitin C-terminal hydrolase-L1 and $\alpha$ II-spectrin breakdown product $145 \mathrm{kDa}$ correlate with outcome after pediatric TBI. J Neurotrauma 2012;29:162-7.

20. Berger RP, Hymel K, Gao WM. The use of biomarkers after inflicted traumatic brain injury: insight into etiology, pathophysiology, and biochemistry. Clinical Pediatric Emergency Medicine 2006;7:186-93.

21. Gao WM, Chadha MS, Berger RP, et al. A gel-based proteomic comparison of human cerebrospinal fluid between inflicted and non-inflicted pediatric traumatic brain injury. J Neurotrauma 2007;24:43-53.

22. Bruun CF, Sanchez JC, Hochstrasser DF, Marhaug G, Husby G. A twodimensional electrophoretic study of serum amyloid A and C-reactive protein in infants and children. Electrophoresis 1998;19:776-81.

23. Woodcock T, Morganti-Kossmann MC. The role of markers of inflammation in traumatic brain injury. Front Neurol 2013;4:18.

24. Rallidis LS, Vikelis M, Panagiotakos DB, et al. Inflammatory markers and in-hospital mortality in acute ischaemic stroke. Atherosclerosis 2006;189:193-7.

25. Hergenroeder G, Redell JB, Moore AN, et al. Identification of serum biomarkers in brain-injured adults: potential for predicting elevated intracranial pressure. J Neurotrauma 2008;25:79-93.

26. Aly H, Hamed Z, Mohsen L, Ramy N, Arnaoot H, Lotfy A. Serum amyloid A protein and hypoxic ischemic encephalopathy in the newborn. J Perinatol 2011;31:263-8.

27. Gerber P, Coffman K. Nonaccidental head trauma in infants. Childs Nerv Syst 2007;23:499-507.

28. Kemp PJ, Searle GJ, Hartness ME, et al. Acute oxygen sensing in cellular models: relevance to the physiology of pulmonary neuroepithelial and carotid bodies. Anat Rec A Discov Mol Cell Evol Biol 2003;270:41-50.

29. Johnson DL, Boal D, Baule R. Role of apnea in nonaccidental head injury. Pediatr Neurosurg 1995;23:305-10.
30. Geddes JF, Hackshaw AK, Vowles GH, Nickols CD, Whitwell HL. Neuropathology of inflicted head injury in children. I. Patterns of brain damage. Brain 2001;124(Pt 7):1290-8.

31. Urieli-Shoval S, Linke RP, Matzner Y. Expression and function of serum amyloid A, a major acute-phase protein, in normal and disease states. Curr Opin Hematol 2000;7:64-9.

32. Gao WM, Kuick R, Orchekowski RP, et al. Distinctive serum protein profiles involving abundant proteins in lung cancer patients based upon antibody microarray analysis. BMC Cancer 2005;5:110.

33. Berger RP, Adelson PD, Pierce MC, Dulani T, Cassidy LD, Kochanek PM. Serum neuron-specific enolase, S100B, and myelin basic protein concentrations after inflicted and noninflicted traumatic brain injury in children. J Neurosurg 2005;103:Suppl 1:61-8.

34. Durham SR, Clancy RR, Leuthardt E, et al. CHOP Infant Coma Scale ("Infant Face Scale"): a novel coma scale for children less than two years of age. J Neurotrauma 2000;17:729-37.

35. Berger RP, Pierce MC, Wisniewski SR, Adelson PD, Kochanek PM. Serum S100B concentrations are increased after closed head injury in children: a preliminary study. J Neurotrauma 2002;19:1405-9.

36. Takata S, Wada H, Tamura M, et al. Kinetics of c-reactive protein (CRP) and serum amyloid A protein (SAA) in patients with community-acquired pneumonia (CAP), as presented with biologic half-life times. Biomarkers 2011;16:530-5.

37. Husby G, Natvig JB. A serum component related to nonimmunoglobulin amyloid protein AS, a possible precursor of the fibrils. J Clin Invest 1974;53:1054-61.

38. Benson MD, Skinner M, Lian J, Cohen AS. "A" protein of amyloidosis. Isolation of a cross-reacting component from serum by affinity chromatography. Arthritis Rheum 1975;18:315-22.

39. Rosenthal CJ, Franklin EC. Variation with age and disease of an amyloid A protein-related serum component. J Clin Invest 1975;55:746-53.

40. Hijmans W, Sipe JD. Levels of the serum amyloid A protein (SAA) in normal persons of different age groups. Clin Exp Immunol 1979;35: 96-100.

41. Jylhävä J, Haarala A, Eklund C, et al. Serum amyloid A is independently associated with metabolic risk factors but not with early atherosclerosis: the Cardiovascular Risk in Young Finns Study. J Intern Med 2009;266:28695.

42. Duffy SO, Squires J, Fromkin JB, Berger RP. Use of skeletal surveys to evaluate for physical abuse: analysis of 703 consecutive skeletal surveys. Pediatrics 2011;127:e47-52.

43. Lu C, Shao C, Cobos E, Singh KP, Gao W. Chemotherapeutic sensitization of leptomycin B resistant lung cancer cells by pretreatment with doxorubicin. PLoS One 2012;7:e32895.

44. Lu C, Zhu W, Shen CL, Gao W. Green tea polyphenols reduce body weight in rats by modulating obesity-related genes. PLoS One 2012;7: e38332. 